\title{
Thermal dynamics of 2-axis electrothermal MEMS micromirrors
}

\author{
Mark Donnachie, Deepak Uttamchandani, and Ralf Bauer \\ University of Strathclyde, Glasgow, United Kingdom \\ mark.donnachie@strath.ac.uk
}

\begin{abstract}
We present the dynamic analysis of a 2 -axis electrothermal MEMS scanner, focusing on the step response times for random access imaging. The $1.2 \mathrm{~mm}$ diameter single layer silicon mirror shows rise times in the $10-40 \mathrm{~ms}$ range for angle changes of $0.4^{\circ}-4.7^{\circ}$, while fall times are 5-15 ms for the same range, leading to the potential of advanced optimization path planning.
\end{abstract}

Keywords- electrothermal MEMS scanner, thermal dynamics, 2D micromirror

\section{INTRODUCTION}

MEMS micromirrors have proven to be a versatile active optical element for miniaturisation of systems ranging from micro-projectors to a host of biomedical endoscopic imaging demonstrations. The most commonly used actuation mechanisms of electrostatic or electromagnetic actuators have shown high angular movement capability and a frequency range in the tens of $\mathrm{kHz}$, but require high voltages or bulky external magnets $[1,2]$. Electrothermal actuation, on the other hand, allows for low voltage driving with the possibility of using simple fabrication techniques. Scanners with high angular range and improved resonance frequency have been demonstrated [3, 4]. These have been integrated in applications ranging from laser engineering to biomedical imaging $[5,6]$. While thermal actuation has an inherently slower response than other techniques due to thermal time constants, the advantage of easy static tilt angle addressing and $2 \mathrm{D}$ operation makes them an interesting prospect for full random access imaging.

In this paper we present the investigation of thermal dynamics of a single device layer 2D electrothermal scanner, with specific interest in the full range of response times to create a dataset for path planning and optimised addressing of random locations in the 2D mirror field of view.

\section{MEMS DESIGN AND SIMULATION}

The design of the $2 \mathrm{D}$ electrothermal micromirror is shown in Fig. 1 (a), consisting of a $1.2 \mathrm{~mm}$ circular mirror surface with four electrothermal actuators distributed around its circumference. The actuators consist of three silicon beams, cross-connected at the top, with $30 \mu \mathrm{m}$ width and length of $1700 \mu \mathrm{m}$ for the two actuators of one main movement axis, and $1800 \mu \mathrm{m}$ for the other. The split in actuator length for the two axes was designed to avoid cross-talk at resonance. The actuators were connected to the mirror surface using serpentine springs. Fabrication of the devices uses Memscap Inc.'s SOIMUMPs multi-user silicon-on-insulator process, with a $10 \mu \mathrm{m}$ thick single crystal silicon device layer and all functional and structural parts designed in it. A $400 \mu \mathrm{m}$ silicon handle wafer is fully back-etched to allow release of the device structure.

This work was supported by the Royal Academy of Engineering under the Engineering for Development Research Fellowship scheme (RF1516 11518) and by an EPSRC Doctoral Training Partnership (EP/R513349/1).

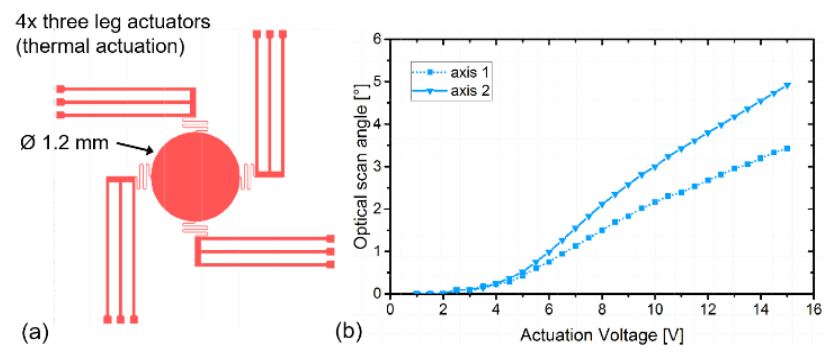

Fig. 1: (a) Schematic of thermal MEMS mirror and (b) static angular displacement with DC voltage.

The static and dynamic thermal behaviour of the micromirror was simulated using Comsol Multiphysics 4.2a, allowing for a Joule Heating evaluation of the mirror scan angle and temperature distribution as well as for the dynamic temperature step response change upon application and removal of the voltage control signals. The measured static optical scan angles of the micromirror are shown in Fig. 1 (b), with a full axis 1 optical angle of $9.8^{\circ}$ and axis 2 optical angle of $6.8^{\circ}$ due to the dual actuator per axis configuration.

\section{THERMAL MEASUREMENTS}

Thermal analysis of the MEMS actuators was investigated experimentally and using FEM simulations at steady state actuation points. The thermal images of the device structure were recorded using a FLIR SC7000 series thermal camera with $\mathrm{x} 3$ magnification objective lens. To simultaneously visualise the heated beam temperature and un-heated device structures, a post-processing high dynamic range composition has been employed. The steady state electrothermal actuation at $5 \mathrm{~V}$ shows heating to $120^{\circ} \mathrm{C}$ of the actuator cross-beam centre (Fig. 2(b)). This is matched well by the FEM simulation, showing a cross-beam temperature of $120^{\circ} \mathrm{C}$ (Fig. 2(a)). At actuation voltages beyond $10 \mathrm{~V}$ a mirror surface temperature increase to $\sim 60^{\circ} \mathrm{C}$ was observed, with a gradient of $8^{\circ} \mathrm{C} / \mathrm{mm}$. Localised radiation effects on adjacent actuator surfaces of non-actuated beams were additionally seen, which were however limited to a shift by $\sim 20^{\circ} \mathrm{C}$. This thermal variation could be caused by the MEMS chip being mounted on thermal isolating material. The simulation results deviate

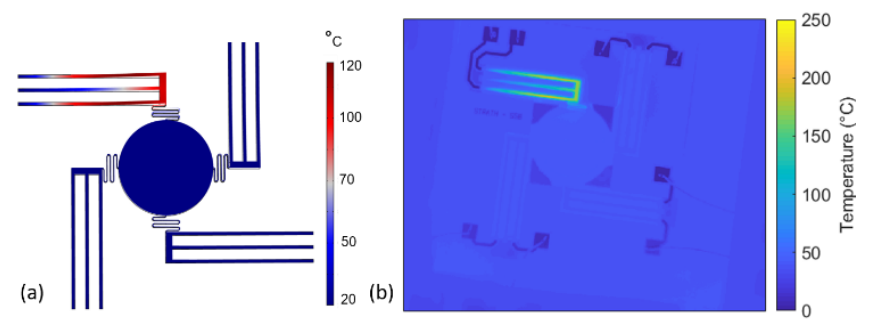

Fig. 2: Static temperature distribution of full mirror for $5 \mathrm{~V}$ actuation on axis 2 actuator; (a) simulated temperature distribution (b) measured temperature distribution. 
from the measured temperature profile at actuation voltages beyond $10 \mathrm{~V}$, which is most likely caused due to uncertainties in the material properties at higher temperatures which are not readily characterised for the used process. The general behaviour and trend is however well matched.

\section{DYNAMIC RESPONSE MEASUREMENTS}

To evaluate the dynamic step response of the electrothermal scanner, a setup consisting of a HeNe laser beam deflected from the MEMS onto a quadrant photodiode (Centronic, QD7-5T) for optical pointing stability and dynamic scan angle evaluation has been built. Automated actuation and data collection is enabled through an Arduino microcontroller combined with custom amplifier boards and LabVIEW integration. The quadrant diode has been mounted on a linear translation stage to allow automated evaluation of mirror step angles moving the reflected HeNe beam beyond the quadrant diode detection area with high precision. The photodiode step response was recorded using a Rigol DS1054Z Oscilloscope, with two halves of the quadrant detector time response recorded with $400 \mathrm{~ns}$ resolution and position changes determined using a custom post-processing Matlab script. Both rise time and fall time measurements have been taken for actuation steps ranging from the maximum mirror response at $14.4 \mathrm{~V}$ to $1 \mathrm{~V}$ steps, representing a static MEMS angle change of $4.7^{\circ}$ and $0.4^{\circ}$ respectively.

The step response for an actuation from $0 \mathrm{~V}$ to $5.3 \mathrm{~V}$ and $14.4 \mathrm{~V}$ is shown in Fig. 3, with a resulting actuator temperature change of $100^{\circ} \mathrm{C}$ and $600^{\circ} \mathrm{C}$ respectively. Both actuation ranges show a significantly faster fall time than rise time, with settling times of $6 \mathrm{~ms}$ and $35 \mathrm{~ms}$ for the rise time and $3 \mathrm{~ms}$ and $7 \mathrm{~ms}$ for the fall time. Larger angular steps show an initial response of the quadrant diode where the reflected laser spot is not present on the active area of the diode, resulting in a fast step down or up of the response, for rise and fall times respectively. An overshoot at higher angular steps shows the mirror to be a minimally underdamped oscillator. The simulated step response times (Fig. 4) show a similar behaviour, with simulated temperature rise times in the 10$40 \mathrm{~ms}$ range while fall times are between 5-15 ms. It should be noted that a discernible movement response of the mirror is only visible above $60^{\circ} \mathrm{C}$, which reduces the fall time. Fig. 5 shows the representation of step response rise and fall times from a variety of initial voltage levels and corresponding MEMS angles. Along the zero line of the $y$ - and $x$-axis are the step response values from and to the rest position,
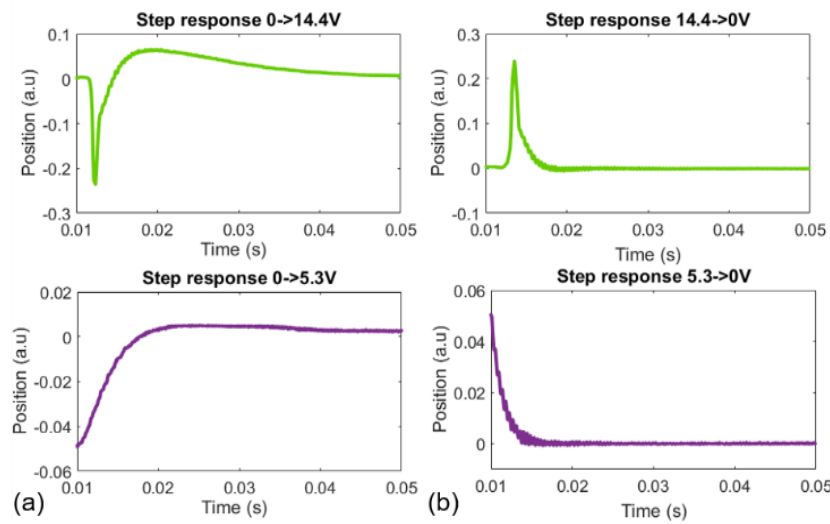

Fig. 3: Measured optical step response time for $0.6^{\circ}$ steps (bottom) and $5.7^{\circ}$ steps (top); (a) rise times show settling times of 10-40 ms (b) fall times show settling times of 5-10 ms.
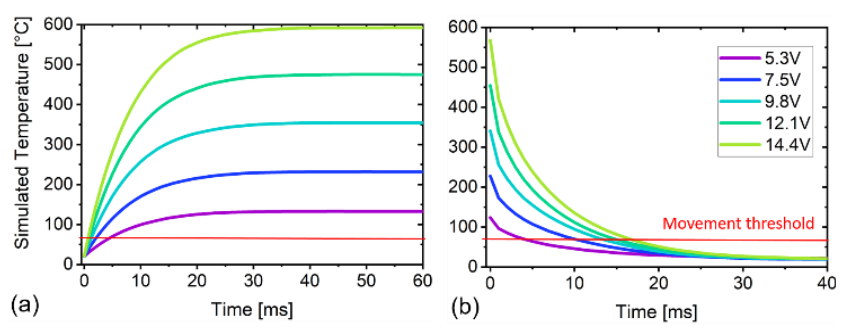

Fig. 4: Thermal step response simulation to estimate rise and fall times of the mirror movement. (a) rise time, (b) fall time; no mirror movement is apparent in the simulation and experiment below $100^{\circ} \mathrm{C}$.

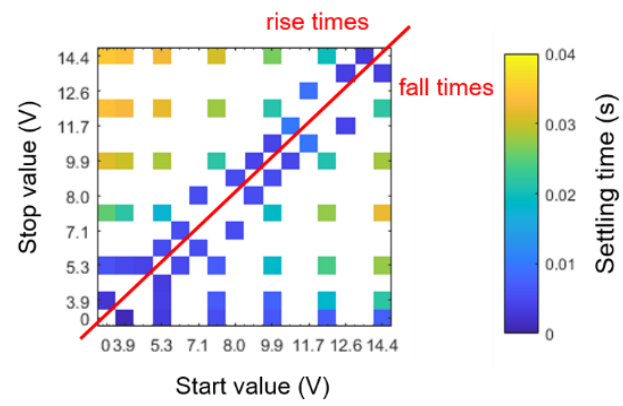

Fig. 5: Measured step response settling time for varying angle changes. The top triangle of the graph represents positive angle steps/rise times, with points close to the diagonal representing small angle steps. The bottom triangle of the graph represents negative angle steps/fall times.

respectively. Response time values for small, $0.4^{\circ}$ steps, along the diagonal of the graph show settling times $<5 \mathrm{~ms}$ while large steps with increasing mirror angle show increasing rise times. For mirror fall times to rest position a faster response is measured, meaning even large angular steps take $<10 \mathrm{~ms}$.

\section{CONCLUSION}

We have shown an in-depth dynamic step response analysis of an electrothermal MEMS scanner, with particular focus on static step response times required for random access imaging applications. A full investigation of the full addressable field-of-view response time for the micromirror, including modelling of the improved fall time movement, will be presented at the conference.

\section{REFERENCES}

[1] A. C. L. Hung, H. Y. H. Lai, T. W. Lin, S. G. Fu, and M. S. C. Lu, "An electrostatically driven 2D micro-scanning mirror with capacitive sensing for projection display," Sensors Actuators, A Phys., vol. 222, pp. 122-129, 2015.

[2] A. R. Cho et al., "Electromagnetic biaxial microscanner with mechanical amplification at resonance," Opt. Express, vol. 23, no. 13, pp. 16792-16802, 2015.

[3] K. Jia, S. Pal, and H. Xie, "An Electrothermal Tip-Tilt-Piston Micromirror Based on Folded Dual S-Shaped Bimorphs," $J$. Microelectromechanical Syst., vol. 18, no. 5, pp. 1004-1015, Oct. 2009.

[4] D. Wang et al., "An ultra-fast electrothermal micromirror with bimorph actuators made of copper/tungsten," Int. Conf. Opt. MEMS Nanophotonics, pp. 4-5, 2017.

[5] A. Paterson, R. Bauer, W. Lubeigt, and D. Uttamchandani, "Tunable Yb:KGW Laser, CW or Q-Switched, Enabled by Dual-Axis Tilt of an MOEMS Mirror," IEEE J. Sel. Top. Quantum Electron., vol. 24, no. 5, p.1601709, Sep. 2018.

[6] C. Duan, Q. Tanguy, A. Pozzi, and H. Xie, "An Optical Coherence Tomography Endoscopic Probe Based on a Tilted MEMS Mirror," Biomed. Opt. 2016, vol. 7, no. 9, p. JW3A.17, 2016. 